University of Wollongong

Research Online

Australian Institute for Innovative Materials -

Papers

Australian Institute for Innovative Materials

$1-1-2019$

Electrocatalytically inactive SnS2 promotes water adsorption/dissociation on molybdenum dichalcogenides for accelerated alkaline hydrogen evolution

Yaping Chen

University of Wollongong, yc463@uowmail.edu.au

Xingyong Wang

University of Wollongong, xingyong@uow.edu.au

Mengmeng Lao

University of Wollongong, ml590@uowmail.edu.au

Kun Rui

University of Wollongong, krui@uow.edu.au

Xiaobo Zheng

University of Wollongong, xz963@uowmail.edu.au

See next page for additional authors

Follow this and additional works at: https://ro.uow.edu.au/aiimpapers

Part of the Engineering Commons, and the Physical Sciences and Mathematics Commons

Research Online is the open access institutional repository for the University of Wollongong. For further information contact the UOW Library: research-pubs@uow.edu.au 


\title{
Electrocatalytically inactive SnS2 promotes water adsorption/dissociation on molybdenum dichalcogenides for accelerated alkaline hydrogen evolution
}

\begin{abstract}
Molybdenum dichalcogenides, in particular, MoS2 and MoSe2, are very promising nonprecious metalbased electrocatalysts for hydrogen evolution reaction (HER) in acidic media. They exhibit inferior alkaline HER activity, however, due to the sluggish water dissociation process. Here, we design and synthesize new molybdenum dichalcogenide-based heterostructures with the basal planes decorated with SnS2 quantum dots towards enhanced alkaline HER activity. The electrochemical results reveal that the alkaline hydrogen evolution kinetics of molybdenum dichalcogenides is substantially accelerated after incorporation of SnS2 quantum dots. The optimal MoSe2/SnS2 heterostructure delivers a much lower overpotential of $285 \mathrm{mV}$ than MoSe2 $(367 \mathrm{mV})$ to reach a current density of $10 \mathrm{~mA} \mathrm{~cm}-2$ in $1 \mathrm{M} \mathrm{KOH}$. The improved catalytic activity is predominantly owing to the enhanced water dissociation kinetics of the heterostructures with well-defined interfaces. Density functional theory (DFT) calculations reveal that the presence of SnS2 significantly promotes the water adsorption capability of MoSe2 nanosheets, which consequently facilitates the subsequent water dissociation process. These results open up a new avenue for the rational design of well-defined heterostructures with enhanced water adsorption/dissociation capability for the development of high-performance alkaline HER electrocatalysts.
\end{abstract}

\section{Keywords}

alkaline, accelerated, dichalcogenides, molybdenum, adsorption/dissociation, hydrogen, water, evolution, promotes, sns2, inactive, electrocatalytically

\section{Disciplines}

Engineering | Physical Sciences and Mathematics

\section{Publication Details}

Chen, Y., Wang, X., Lao, M., Rui, K., Zheng, X., Yu, H., Ma, J., Dou, S. Xue. \& Sun, W. (2019).

Electrocatalytically inactive SnS2 promotes water adsorption/dissociation on molybdenum dichalcogenides for accelerated alkaline hydrogen evolution. Nano Energy, 64 1-6.

\section{Authors}

Yaping Chen, Xingyong Wang, Mengmeng Lao, Kun Rui, Xiaobo Zheng, Haibo Yu, Jing Ma, Shi Xue Dou, and Wenping Sun 
Electrocatalytically Inactive $\mathrm{SnS}_{2}$ Promotes Water Adsorption/Dissociation on Molybdenum Dichalcogenides for Accelerated Alkaline Hydrogen Evolution

Yaping Chen ${ }^{a,+}$, Xingyong Wang ${ }^{b,+}$, Mengmeng Lao ${ }^{a}, K_{u n}$ Rui $^{a}$, Xiaobo Zheng ${ }^{a}$, Haibo Yu ${ }^{b, *}$, Jing Ma ${ }^{c}$, Shi Xue Dou ${ }^{a}$, Wenping Sun ${ }^{a}$ *

${ }^{a}$ Institute for Superconducting and Electronic Materials, Australian Institute for Innovative Materials, University of Wollongong, Wollongong, NSW 2522, Australia

b School of Chemistry and Molecular Bioscience, University of Wollongong, Wollongong, NSW 2522, Australia

${ }^{c}$ School of Chemistry and Chemical Engineering, Institute of Theoretical and Computational Chemistry, Key Laboratory of Mesoscopic Chemistry of the Ministry of Education (MOE), Nanjing University, Nanjing 210093, P. R. China

* Corresponding author.

E-mail address: hyu@uow.edu.au; wenping@uow.edu.au

+ These authors contributed equally to this work

\section{Abstract}

Molybdenum dichalcogenides, in particular, $\mathrm{MoS}_{2}$ and $\mathrm{MoSe}_{2}$, are very promising nonprecious metal-based electrocatalysts for hydrogen evolution reaction (HER) in acidic media. They exhibit inferior alkaline HER activity, however, due to the sluggish water dissociation process. Here, we design and synthesize new molybdenum dichalcogenide-based heterostructures with the basal planes decorated with $\mathrm{SnS}_{2}$ quantum dots towards enhanced alkaline HER activity. The electrochemical results reveal that the alkaline hydrogen evolution kinetics of molybdenum dichalcogenides is substantially accelerated after incorporation of $\mathrm{SnS}_{2}$ quantum dots. The optimal $\mathrm{MoSe}_{2} / \mathrm{SnS}_{2}$ heterostructure delivers a much lower overpotential of $285 \mathrm{mV}$ than $\mathrm{MoSe}_{2}(367 \mathrm{mV})$ to reach a current density of $10 \mathrm{~mA} \mathrm{~cm}{ }^{-2}$ in $1 \mathrm{M} \mathrm{KOH}$. The improved catalytic activity is predominantly owing to the enhanced water dissociation kinetics of the heterostructures with well-defined interfaces. Density functional theory (DFT) calculations reveal that the presence of $\mathrm{SnS}_{2}$ significantly promotes the water adsorption capability of $\mathrm{MoSe}_{2}$ nanosheets, which consequently facilitates the subsequent water dissociation process. These results open up a new avenue for the rational design of well-defined heterostructures 
with enhanced water adsorption/dissociation capability for the development of highperformance alkaline HER electrocatalysts.

Keywords: Molybdenum dichalcogenides; $\mathrm{SnS}_{2}$; heterostructure; water adsorption; hydrogen evolution reaction

\section{Introduction}

Our severe energy and environmental crisis makes it imperative to search for clean and sustainable energy sources as alternatives to traditional fossil fuels. ${ }^{[1-3]}$ Owing to its having the highest gravimetric energy density and carbon-free emissions, hydrogen produced by renewable energy sources is considered to be the most promising energy carrier for our future society's energy. ${ }^{[4-5]}$ Currently, hydrogen is mostly produced from fossil fuels by steam reforming. ${ }^{[6]}$ Alternatively, photocatalytic, photoelectrocatalytic, or electrocatalytic water splitting driven by renewable energy would make hydrogen a real carrier for clean energy. ${ }^{[7-9]}$ With regards to electrocatalytic water splitting, electrocatalytic performance remains unsatisfactory for both the cathodic hydrogen evolution reaction (HER) and the anodic oxygen evolution reaction (OER), although numerous research efforts have been devoted to developing efficient electrocatalysts. Currently, precious metal-based materials are the state-of-the-art catalysts for both the HER (e.g., Pt) and the OER (e.g., $\mathrm{IrO}_{2}$ ), but they suffer from high cost and scarcity. ${ }^{[10-17]}$ Therefore, developing earth-abundant and low-cost alternatives, such as transition metal chalcogenides, metal oxides/hydroxides, and metal alloys, is critically necessary to address this challenge for practical water splitting systems. ${ }^{[18-21]}$

Molybdenum dichalcogenides, in particular $\mathrm{MoS}_{2}$ and $\mathrm{MoSe}_{2}$, are very promising nonpreciousmetal-based electrocatalysts for the HER. ${ }^{[22-24]}$ Both density functional theory (DFT) calculations and experimental findings have demonstrated that the HER catalytic activity of molybdenum dichalcogenides is mainly derived from their edge sites. ${ }^{[25-29]}$ In this regards, various strategies have been focused on increasing the number of exposed active sites of 
molybdenum-dichalcogenide-based electrocatalysts through building various nanostructures, engineering surface defects, or heteroatom doping. ${ }^{[30-32]}$ Unfortunately, although the molybdenum dichalcogenide-based electrocatalysts thus developed display impressive catalytic activity in acidic media, they exhibit inferior HER activity in alkaline media due to the sluggish water dissociation kinetics. Basically, alkaline HER involves water adsorption, water dissociation, and hydrogen recombination and release. ${ }^{[33]}$ Water adsorption and dissociation take place at the beginning of the alkaline HER process and are considered to represent the rate-determining step for the alkaline HER. ${ }^{[34-37]}$ Therefore, designing electrocatalysts with enhanced water adsorption and dissociation capability is the key for the promotion of alkaline HER catalytic activity. Recently, molybdenum-dichalcogenide-based heterostructures with an additional phase (e.g., $\mathrm{Ni}(\mathrm{OH})_{2}$ ) anchored on $\mathrm{MoS}_{2}$ or $\mathrm{MoSe}_{2}$ nanosheets were reported as efficient alkaline HER catalysts. ${ }^{[38-40]}$ The second phases usually possess strong water affinity and water adsorption capability, which are of great significance for accelerating the water dissociation kinetics of the heterostructured catalysts. ${ }^{[41]}$ Meanwhile, in some cases, the presence of the second phase can also modulate the electronic structure of Mo and optimize the hydrogen adsorption energy.$^{[42-44]}$ On the other hand, heteroatom doping (e.g., Ni, Co) was also demonstrated to be an effective strategy for enhancing alkaline HER kinetics. ${ }^{[45-46]}$

In this work, we propose a new heterostructured design concept in order to improve the alkaline HER activity of molybdenum dichalcogenides. $\mathrm{MoSe}_{2} / \mathrm{SnS}_{2}$ and $\mathrm{MoS}_{2} / \mathrm{SnS}_{2}$ heterostructures with $\mathrm{SnS}_{2}$ quantum dots decorated on the basal planes are synthesized by a universal wetchemical strategy for enhanced alkaline HER. DFT calculations reveal that the incorporation of $\mathrm{SnS}_{2}$ brings in the substantial enhancement of water adsorption capability of $\mathrm{MoSe}_{2}$ both on the edge sites and basal planes. Benefiting from the improved water adsorption/dissociation 
capability, the well-defined heterostructures delivered significantly enhanced hydrogen evolution kinetics in alkaline media.

\section{Experiment section}

\subsection{Materials synthesis}

Synthesis of $\mathrm{MoSe}_{2}$ nanosheets. MoSe 2 nanosheets were synthesized by a modified hydrothermal process. ${ }^{[32]}$ Briefly, $241.95 \mathrm{mg} \mathrm{Na} 2 \mathrm{MoO}_{4} \cdot 2 \mathrm{H}_{2} \mathrm{O}$ was added to $20 \mathrm{ml}$ deionized water (DI water) under magnetic stirring as the Mo precursor. Then, $0.1 \mathrm{~g} \mathrm{NaBH}_{4}$ was dissolved in $15 \mathrm{ml}$ Ar saturated DI water in a three-neck bottle. Subsequently, $0.16 \mathrm{~g}$ Se powders were dispersed into the $\mathrm{NaBH}_{4}$ aqueous solution under Ar flow with mild shaking until Se powders were fully dissolved to form a homogeneous transparent NaHSe solution, which was used as the Se source. Next, the Mo and Se source solutions were transferred into a $50 \mathrm{ml}$ Teflon-lined stainless steel autoclave, purged with Ar for $30 \mathrm{~min}$, and then heated in an oven at $180{ }^{\circ} \mathrm{C}$ for 24 h. After cooling down to room temperature, the as-prepared product was collected by centrifugation, washed with DI water and ethanol for several times, and dried at $60{ }^{\circ} \mathrm{C}$ under vacuum overnight.

Synthesis of $\mathrm{MoSe}_{2} / \mathrm{SnS}_{2}$ heterostructures. For the preparation of $\mathrm{MoSe}_{2} / \mathrm{SnS}_{2}-2.5,7.12 \mathrm{mg}$ $\mathrm{SnCl}_{4} \cdot 5 \mathrm{H}_{2} \mathrm{O}$ and $6 \mathrm{mg}$ thioacetamide (TAA) were added together into the as-obtained $\mathrm{MoSe}_{2}$ dispersion solution $\left(0.36 \mathrm{mg} \mathrm{ml}^{-1}\right.$ in the mixture of $15 \mathrm{ml}$ DI water and $20 \mathrm{ml}$ ethylene glycol). After $1 \mathrm{~h}$ sonication, the mixed solution was transferred into a $50 \mathrm{ml}$ Teflon-lined stainless steel autoclave and heated in an electric oven at $180{ }^{\circ} \mathrm{C}$ for $24 \mathrm{~h}$. After cooling down to room temperature, the obtained $\mathrm{MoSe}_{2} / \mathrm{SnS}_{2}-2.5$ was collected by centrifugation at $8000 \mathrm{rpm}$ for 5 min, then repeatedly washed with DI water and ethanol, and finally dried at $60{ }^{\circ} \mathrm{C}$ under vacuum overnight. $\mathrm{MoSe}_{2} / \mathrm{SnS}_{2}-x(x=1.5,5.0,10)$ was prepared following the same procedure as for $\mathrm{MoSe}_{2} / \mathrm{SnS}_{2}-2.5$ with different molar ratios of Mo and $\mathrm{Sn}$ precursors $(\mathrm{Mo} / \mathrm{Sn}=1.5,5.0$, 
and 10). The $\mathrm{Mo} / \mathrm{Sn}$ atomic ratios in the $\mathrm{MoSe}_{2} / \mathrm{SnS}_{2}$ heterostructures $\left(\mathrm{MoSe}_{2} / \mathrm{SnS}_{2}-1.5: \mathrm{Mo} / \mathrm{Sn}\right.$ = 3.5; $\mathrm{MoSe}_{2} / \mathrm{SnS}_{2}-2.5: \mathrm{Mo} / \mathrm{Sn}=4.7 ; \mathrm{MoSe}_{2} / \mathrm{SnS}_{2}-5.0: \mathrm{Mo} / \mathrm{Sn}=7.0 ; \mathrm{MoSe}_{2} / \mathrm{SnS}_{2}-10: \mathrm{Mo} / \mathrm{Sn}$ $=12.6)$ were determined using inductively coupled plasma - optical emission spectrometry (ICP-OES, Perkin-Elmer, Optima 7300DV) with mass spectrometry.

Synthesis of $\mathrm{MoS}_{2} / \mathrm{SnS} \mathrm{S}_{2}$ heterostructures. For the preparation of pure $\mathrm{MoS}_{2}, 72.6 \mathrm{mg} \mathrm{Na} 2 \mathrm{MoO}_{4}$ $2 \mathrm{H}_{2} \mathrm{O}$ and $45.7 \mathrm{mg}$ thiourea were firstly added into DI water $(35 \mathrm{ml})$ under sonication for 10 mins. Then, the homogeneous solution was transferred into a $50 \mathrm{~mL}$ autoclave for hydrothermal reaction at $180{ }^{\circ} \mathrm{C}$ for $24 \mathrm{~h}$. After cooling down to room temperature, the as-prepared $\mathrm{MoS}_{2}$ was collected by centrifugation, washed with DI water and ethanol several times, and dried at $60{ }^{\circ} \mathrm{C}$ under vacuum overnight. For the preparation of $\mathrm{MoS}_{2} / \mathrm{SnS}_{2}-2.5,7.12 \mathrm{mg} \mathrm{SnCl} \mathrm{Sn}_{4} \cdot 5 \mathrm{H}_{2} \mathrm{O}$ and $6 \mathrm{mg}$ TAA were added together into the as-obtained $\mathrm{MoS}_{2}$ dispersion solution $(0.232 \mathrm{mg}$ $\mathrm{ml}^{-1}$ in the mixture of $15 \mathrm{ml} \mathrm{DI}$ water and $20 \mathrm{ml}$ ethylene glycol). After $1 \mathrm{~h}$ sonication, the mixed solution was transferred into the $50 \mathrm{ml}$ Teflon-lined stainless steel autoclave and heated in an electric oven at $180{ }^{\circ} \mathrm{C}$ for $24 \mathrm{~h}$. Finally, the $\mathrm{MoS}_{2} / \mathrm{SnS}_{2}-2.5$ product was collected after centrifugation, washing, and drying. $\mathrm{MoS}_{2} / \mathrm{SnS}_{2}-x(x=1.5$ and 5.0) was prepared following the same procedure as for $\mathrm{MoS}_{2} / \mathrm{SnS}_{2}-2.5$ with different molar ratios of Mo and $\mathrm{Sn}$ precursors $(\mathrm{Mo} / \mathrm{Sn}=1.5$ and 5.0)

\subsection{Physical Characterization}

X-ray diffraction (XRD) was carried out using a GBC MMA X-ray diffractometer $(\lambda=1.5406$ $\AA$ A $25 \mathrm{~mA}, 40 \mathrm{Kv}$, step size of $0.02^{\circ} \mathrm{s}^{-1}$ ). The morphology and microstructures of the samples were characterized by transmission electron microscopy (TEM, JEM-2010, working voltage $200 \mathrm{kV}$ ). The scanning transmission electron microscopy (STEM) elemental mappings were conducted on the JEOL ARM-200F at $200 \mathrm{kV}$ equipped with an EDS detector. X-ray photoelectron spectroscopy (XPS) measurements were performed on a Thermo ESCALAB 
250Xi instrument with monochrome $\mathrm{Al} \mathrm{K} \alpha(h v=1486.6 \mathrm{eV})$ as the $\mathrm{X}$-ray excitation source. The $\mathrm{Mo} / \mathrm{Sn}$ atomic ratios in the $\mathrm{MoSe}_{2} / \mathrm{SnS}_{2}$ heterostructures were obtained by ICP-OES.

\subsection{Electrochemical measurements}

All the electrochemical measurements were performed with a typical three-electrode electrochemical cell equipped with the rotating disk electrode (Pine Research Instruments, Inc.) and electrochemical workstation (Multichannel potentiostat/galvanostat VSP-300, BioLogic Science Instrument). $\mathrm{Hg} / \mathrm{HgO}$ (1 $\mathrm{M} \mathrm{KOH}$ solution) and $\mathrm{Ag} / \mathrm{AgCl}$ (saturated $\mathrm{KCl}$ solution) were used as the reference electrode in $1 \mathrm{M} \mathrm{KOH}$ and $0.5 \mathrm{M} \mathrm{H}_{2} \mathrm{SO}_{4}$ aqueous solution, respectively. The platinum wire was employed as the counter electrode, and the glassy carbon electrode $\left(0.196 \mathrm{~cm}^{2}\right)$ coated with different catalyst inks was used as the working electrode. The catalyst inks were prepared as follows. $2 \mathrm{mg}$ catalyst was dispersed into a mixed solvent composed of $16 \mu \mathrm{L}$ Nafion solution (Aldrich Co., $5 \mathrm{wt} \%$ ), $384 \mu \mathrm{L}$ deionized water, and $100 \mu \mathrm{L}$ isopropanol under sonication for $30 \mathrm{~min} .10 \mu \mathrm{L}$ of catalyst ink (containing $40 \mu \mathrm{g}$ of catalyst) was coated onto the polished glassy carbon electrode and dried at room temperature. The electrolyte was continuously purged with $\mathrm{N}_{2}$ to remove $\mathrm{O}_{2}$ during the measurements. The working electrode was constantly rotated at $1600 \mathrm{rpm}$ to remove the generated $\mathrm{H}_{2}$ and eliminate concentration polarization of electrolyte ions during electrochemical testing. Linear sweep voltammetry (LSV) polarization curves were collected at a scan rate of $5 \mathrm{mV} \mathrm{s}^{-1}$. All LSV curves were corrected with $95 \%$ iR-compensation. Electrochemical impedance spectra (EIS) were collected at $-0.1 \mathrm{~V}$ (vs. RHE) in the frequency range of $1.0-100 \mathrm{kHz}$.

The electrochemical active surface area (ECSA) was evaluated by calculating the double-layer capacitance $\left(C_{\mathrm{dl}}\right)$ in $1 \mathrm{M} \mathrm{KOH} \cdot{ }^{[47]}$ The cyclic voltammetry $(\mathrm{CV})$ curves were performed at scan rates from 40 to $140 \mathrm{mV} \mathrm{s}^{-1}$ in the range of $-100-0 \mathrm{mV}$ vs. RHE. The $C_{\mathrm{dl}}$ values were calculated according to the equation: 


$$
j=v C_{d l}
$$

Where the capacitive current $\mathrm{j}(\mathrm{A})$ can be obtained by $\frac{J_{a}-J_{c}}{2}$. $\mathrm{J}_{\mathrm{a}}$ and $\mathrm{J}_{\mathrm{c}}$ represent anodic current and cathodic current at $-50 \mathrm{mV}$ vs. RHE, respectively, $v\left(\mathrm{mV} \mathrm{s}^{-1}\right)$ is the scan rate.

The turnover frequency (TOF) was calculated according to the following equation:

$$
T O F=\frac{\text { Total number of } \mathrm{H}_{2} \text { molecules per second }}{\text { Total number of active sites per unit area }}=\frac{\frac{j}{2 \times q}}{\mathrm{~N}}
$$

Where $q=1.6 \times 10^{-19} \mathrm{C}$ stands for the elementary charge, $\mathrm{j}$ represents current $(\mathrm{A})$ from the linear sweep measurement, $\mathrm{N}$ stands for the number of total Mo atoms, and 2 represents that two electrons are required to generate one $\mathrm{H}_{2}$ molecule.

\section{Results and discussion}

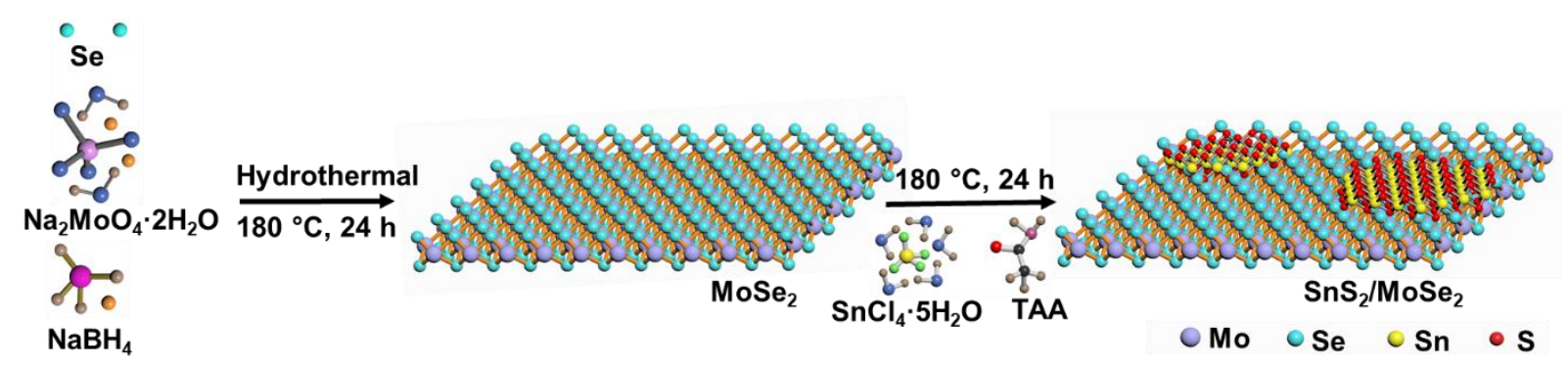

Scheme 1. Illustration of the synthesis of $\mathrm{MoSe}_{2} / \mathrm{SnS}_{2}$ heterostructures.

The molybdenum dichalcogenide heterostructures were prepared via a two-step hydrothermal method, as illustrated in Scheme 1. The molybdenum dichalcogenide nanosheets were first prepared, and then $\mathrm{SnS}_{2}$ quantum dots were uniformly anchored on the nanosheet surfaces via an in situ precipitation process (See Experimental Section for more details). The X-ray diffraction (XRD) pattern of $\mathrm{MoSe}_{2} / \mathrm{SnS}_{2}$ (Figure S1, Supporting Information) presents typical diffraction peaks that can be well indexed to $\mathrm{SnS}_{2}$ (JCPDS No. 23-0667) and $\mathrm{MoSe}_{2}$ (JCPDS No. 29-0914). Figure 1a shows the transmission electron microscopy (TEM) analysis, $\mathrm{SnS}_{2}$ quantum dots with a size of 3-5 nm were uniformly grown on the surfaces of $\mathrm{MoSe}_{2}$ nanosheets, 
which could reduce agglomeration. Also, the typical lattice spacings of 0.32 and $0.26 \mathrm{~nm}$ could be indexed to the (100) planes of $\mathrm{SnS}_{2}$ and the (102) planes of $\mathrm{MoSe}_{2}$, respectively. In Figure 1b, the high-resolution transmission electron microscopy (HRTEM) image provides more detailed structural information. It can be shown that the markedly striped patterns are in good agreement with the $\mathrm{MoSe}_{2}$ edge surface of (002) planes, while the $\mathrm{SnS}_{2}$ quantum dots were aligned along the [001] $c$-axis in intimately contact with the $\mathrm{MoSe}_{2}$ surface. The corresponding Fast Fourier transform (FFT) pattern (Figure 1c) clearly indicates the co-existence of (102) and (002) planes of $\mathrm{MoSe}_{2}$, and (100) and (101) planes of $\mathrm{SnS}_{2}$, further revealing the formation of $\mathrm{MoSe}_{2} / \mathrm{SnS}_{2}$ heterostructures. The scanning transmission electron microscopy (STEM) elemental mapping results further reveal the spatial distribution of Mo, Se, Sn, and $\mathrm{S}$ in the $\mathrm{MoSe}_{2} / \mathrm{SnS}_{2}$ heterostructures (Figure 1d). Moreover, the $\mathrm{MoSe}_{2} / \mathrm{SnS}_{2}$ heterostructures with different $\mathrm{Mo} / \mathrm{Sn}$ atomic ratios could be precisely controlled by varying the ratio of pthe recursors (Figure S2, Supporting Information).

The X-ray photoelectron spectroscopy (XPS) survey spectrum of the $\mathrm{MoSe}_{2} / \mathrm{SnS}_{2}$ heterostructures confirmed the coexistence of Mo, Se, Sn, and S elements (Figure S3, Supporting Information). After deposition of the $\mathrm{SnS}_{2}$, the binding energies of Mo $3 \mathrm{~d}$ and $\mathrm{Se}$ $3 \mathrm{~d}$ are shifted negatively by 0.2 and $0.3 \mathrm{eV}$, respectively (Figure 1e-f). Along with the negative shift in the binding energy, the surface negative charge density of $\mathrm{MoSe}_{2}$ will be increased. As a result, the $\mathrm{H}$ atoms of water molecules are more accessible to the negative charges, so that an attractive interaction between water molecules and the surface of $\mathrm{MoSe}_{2}$ is enhanced accordingly. ${ }^{[26,48]}$ In other words, the negative shift in the binding energy for Mo and Se effectively promotes the adsorption capability of water molecules. Also, the negative shift in the binding energy for $\mathrm{MoSe}_{2}$ demonstrates the transfer of electrons from $\mathrm{SnS}_{2}$ to $\mathrm{MoSe}_{2}$, which helps to improve the conductivity of $\mathrm{MoSe}_{2} \cdot{ }^{[43-44]}$ Moreover, a positive shift of $0.5 \mathrm{eV}$ can be observed for the binding energy of $\mathrm{Sn} 3 \mathrm{~d}$ in $\mathrm{MoSe}_{2} / \mathrm{SnS}_{2}$ as compared to $\mathrm{SnS}_{2}$ (Figure $1 \mathrm{~g}$ ). 
These results demonstrate that the charge redistribution across the interfaces of $\mathrm{MoSe}_{2} / \mathrm{SnS}_{2}$ heterostructures is beneficial to increase the water adsorption capability and improve the conductivity of $\mathrm{MoSe}_{2}$.
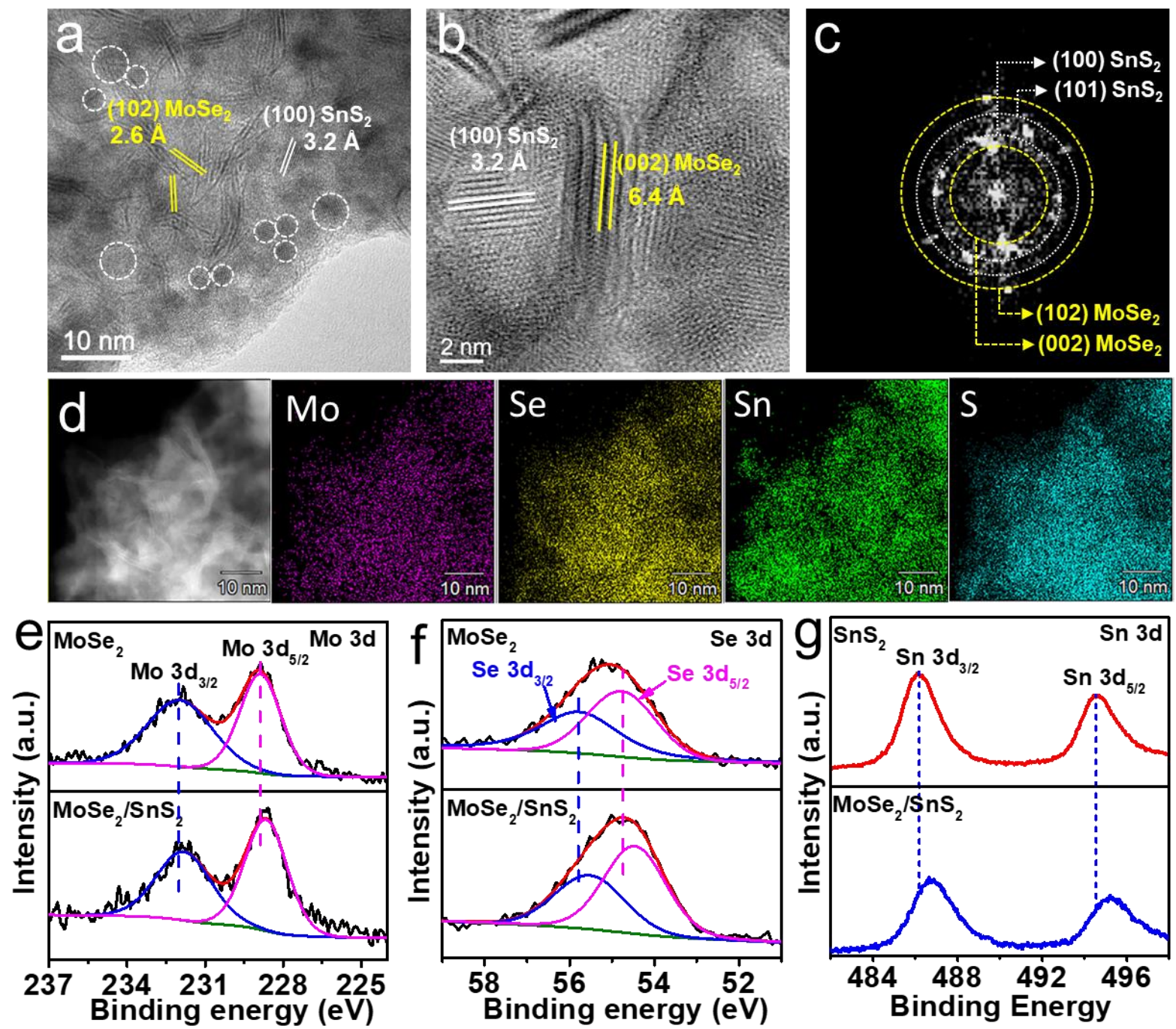

Figure 1. (a, b) TEM images and (c) the corresponding FFT pattern of $\mathrm{MoSe}_{2} / \mathrm{SnS}_{2}-2.5$ heterostructure; (d) STEM-EDS elemental mapping of Mo, Se, Sn, and S; XPS spectra of (e) Mo 3d, (f) Se 3d, and (g) Sn $3 \mathrm{~d}$.

The HER activities of the as-prepared $\mathrm{MoSe}_{2}$ and $\mathrm{MoSe}_{2} / \mathrm{SnS}_{2}$ heterostructured catalysts were evaluated in $1 \mathrm{M} \mathrm{KOH}$ aqueous electrolyte in a standard three-electrode system. Figure 2a shows the polarization curves after $95 \% \mathrm{iR}$ correction carried out at a scan rate of $5 \mathrm{mV} \mathrm{s}^{-1}$. For the pure $\mathrm{SnS}_{2}$, the linear sweep voltammetry (LSV) curve displays a weak current response, demonstrating its electrocatalytic inertness in alkaline media. The pure $\mathrm{MoSe}_{2}$ needs an 
overpotential of $367 \mathrm{mV}$ to reach a current density of $10 \mathrm{~mA} \mathrm{~cm}{ }^{-2}$. Compared to $\mathrm{MoSe}_{2}$, all the $\mathrm{MoSe}_{2} / \mathrm{SnS}_{2}$ heterostructured catalysts show substantially enhanced catalytic activities. Specifically, the $\mathrm{MoSe}_{2} / \mathrm{SnS}_{2}-2.5$ heterostructured catalyst exhibits optimal HER activity and a much lower overpotential of $285 \mathrm{mV}$ at $10 \mathrm{~mA} \mathrm{~cm}^{-2}$ (Figure 2b). Also, $\mathrm{MoSe}_{2} / \mathrm{SnS}_{2}-2.5$ shows the best mass activity (559 $\left.\mathrm{mA} \mathrm{mg}^{-1}\right)$ at $0.45 \mathrm{~V}$ among all the catalysts, which is superior to $\mathrm{MoSe}_{2} / \mathrm{SnS}_{2}-1.5\left(309 \mathrm{~mA} \mathrm{mg}{ }^{-1}\right), \mathrm{MoSe}_{2} / \mathrm{SnS}_{2}-5.0\left(291 \mathrm{~mA} \mathrm{mg}{ }^{-1}\right), \mathrm{MoSe}_{2} / \mathrm{SnS}_{2}-10(235 \mathrm{~mA}$ $\left.\mathrm{mg}^{-1}\right)$, and pure $\mathrm{MoSe}_{2}\left(147 \mathrm{~mA} \mathrm{mg}^{-1}\right.$ ) (Figure 2c and the inset). These results indicate that $\mathrm{SnS}_{2}$ plays a key role in promoting the HER kinetics of $\mathrm{MoSe}_{2}$ in alkaline media. It is worth noting that the physically mixed sample with a molar ratio of $\operatorname{MoSe}_{2}: \mathrm{SnS}_{2}=2.5: 1$ $\left(\mathrm{MoSe}_{2}+\mathrm{SnS}_{2}-2.5\right)$ deliveres decreased geometric and mass activities $\left(14 \mathrm{~mA} \mathrm{~cm}{ }^{-2}\right.$ and $87 \mathrm{~mA}$ $\mathrm{mg}^{-1}$ at $0.45 \mathrm{~V}$ ), as compared to $\mathrm{MoSe}_{2} / \mathrm{SnS}_{2}-2.5$ and bare $\mathrm{MoSe}_{2}$ (Figure S4, Supporting Information), revealing that the unique heterostructure morphology and interaction between $\mathrm{MoSe}_{2}$ and $\mathrm{SnS}_{2}$ are of great importance to the accelerated alkaline HER kinetics. Tafel slopes give further insights into the HER kinetics. As shown in Figure 2d, pure $\mathrm{MoSe}_{2}$ shows a Tafel slope of $149 \mathrm{mV} \mathrm{dec}^{-1}$, indicating that the kinetic rate-limiting step is the Volmer step, in which step water molecules dissociate into hydrogen intermediates and hydroxyls. Compared to $\mathrm{MoSe}_{2}$, the Tafel slope of $\mathrm{MoSe}_{2} / \mathrm{SnS}_{2}-2.5$ is reduced to $109 \mathrm{mV} \mathrm{dec}^{-1}$, demonstrating that the HER kinetics are determined by the Volmer step and subsequent Heyrovsky step. The enhanced kinetics can be attributed to the accelerated water dissociation process (Volmer step). ${ }^{[45]}$ 

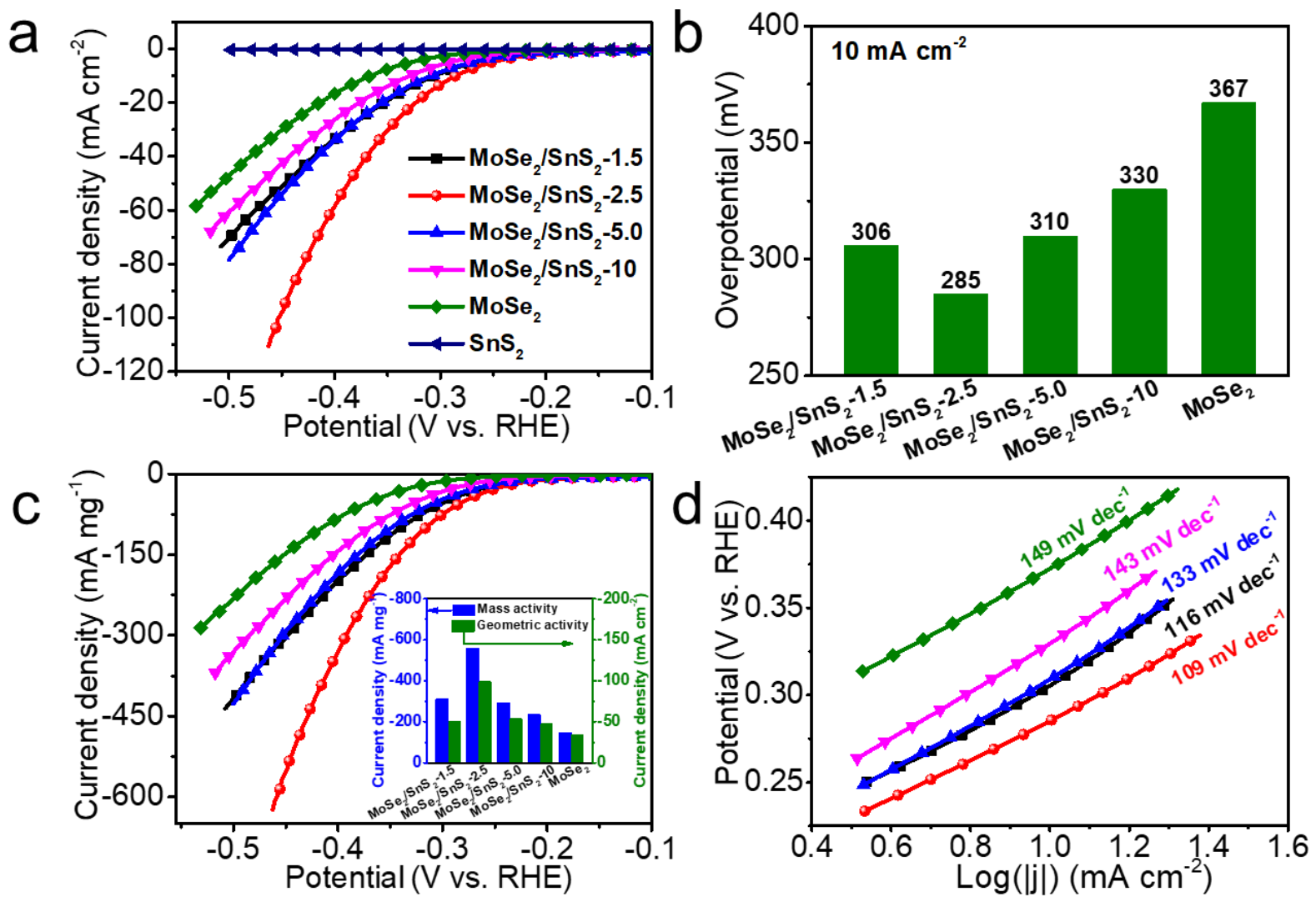

Figure 2. (a) LSV polarization curves measured at a scan rate of $5 \mathrm{mV} \mathrm{s}^{-1}$; (b) Overpotential at the current density of $10 \mathrm{~mA} \mathrm{~cm}^{-2}$; (c) LSV curves plotted based on $\mathrm{MoSe}_{2}$ mass-normalized current density (inset: current densities at $-0.45 \mathrm{~V}$ (vs. RHE) ); (d) Tafel plots (potential vs. $\log$ (current density)) derived from LSV curves.

Turnover frequencies (TOFs) provide important insights in evaluating the intrinsic activity of HER catalysts. Generally, it is supposed that TOFs reflect the formation rate of hydrogen molecules per Mo atom during the HER process. ${ }^{[49-50]}$ Here, we identify the TOF value of $\mathrm{MoSe}_{2} / \mathrm{SnS}_{2}$ catalysts based on $\mathrm{MoSe}_{2}$ which provides intrinsic active sites for the HER. Although the TOF calculation here employs the total Mo atoms in the electrode as the benchmark, which may not exactly reflect the performance of the $\mathrm{MoSe}_{2} / \mathrm{SnS}_{2}$ heterostructures, it provides a general comparison of the HER kinetics between $\mathrm{MoSe}_{2}$ and $\mathrm{MoSe}_{2} / \mathrm{SnS}_{2}$ composites. The $\mathrm{MoSe}_{2} / \mathrm{SnS}_{2}-2.5$ achieves the highest TOF as compared with other samples (Figure 3a). Meanwhile, the electrochemically active surface area (ECSA), as another critical factor for estimating the activity of electrocatalysts, was determined by measuring the doublelayer capacitance $\left(C_{\mathrm{dl}}\right)$ in the potential range of $-0.1-0 \mathrm{~V}$ (vs. RHE) (Figure S5, Supporting 
Information). As shown in Figure 3b, all the $\mathrm{MoSe}_{2} / \mathrm{SnS}_{2}$ heterostructured catalysts display larger $C_{\mathrm{dl}}$ values than that of pure $\mathrm{MoSe}_{2}$, indicating that the introduction of $\mathrm{SnS}_{2}$ endows the heterostructured catalysts with higher active site density. ${ }^{[51]}$ Moreover, the enhanced catalytic activity of $\mathrm{MoSe}_{2} / \mathrm{SnS}_{2}$ heterostructures was confirmed by electrochemical impedance spectroscopy (EIS). The Nyquist plots are well fitted to an equivalent circuit model (Figure S6, Supporting Information), which consists of electrolyte resistance $\left(R_{\mathrm{S}}\right)$, charge-transfer resistance $\left(R_{\mathrm{ct}}\right)$, and constant phase element $(\mathrm{CPE})$. All the heterostructured catalysts show much lower $\mathrm{R}_{\mathrm{ct}}$ than that of bare $\mathrm{MoSe}_{2}$ (Table $\mathrm{S} 2$, Supporting Information), further demonstrating that the incorporation of $\mathrm{SnS}_{2}$ is beneficial for accelerating the charge transfer and mass diffusion kinetics of $\mathrm{MoSe}_{2} / \mathrm{SnS}_{2}$ heterostructures under alkaline conditions. ${ }^{[52]}$
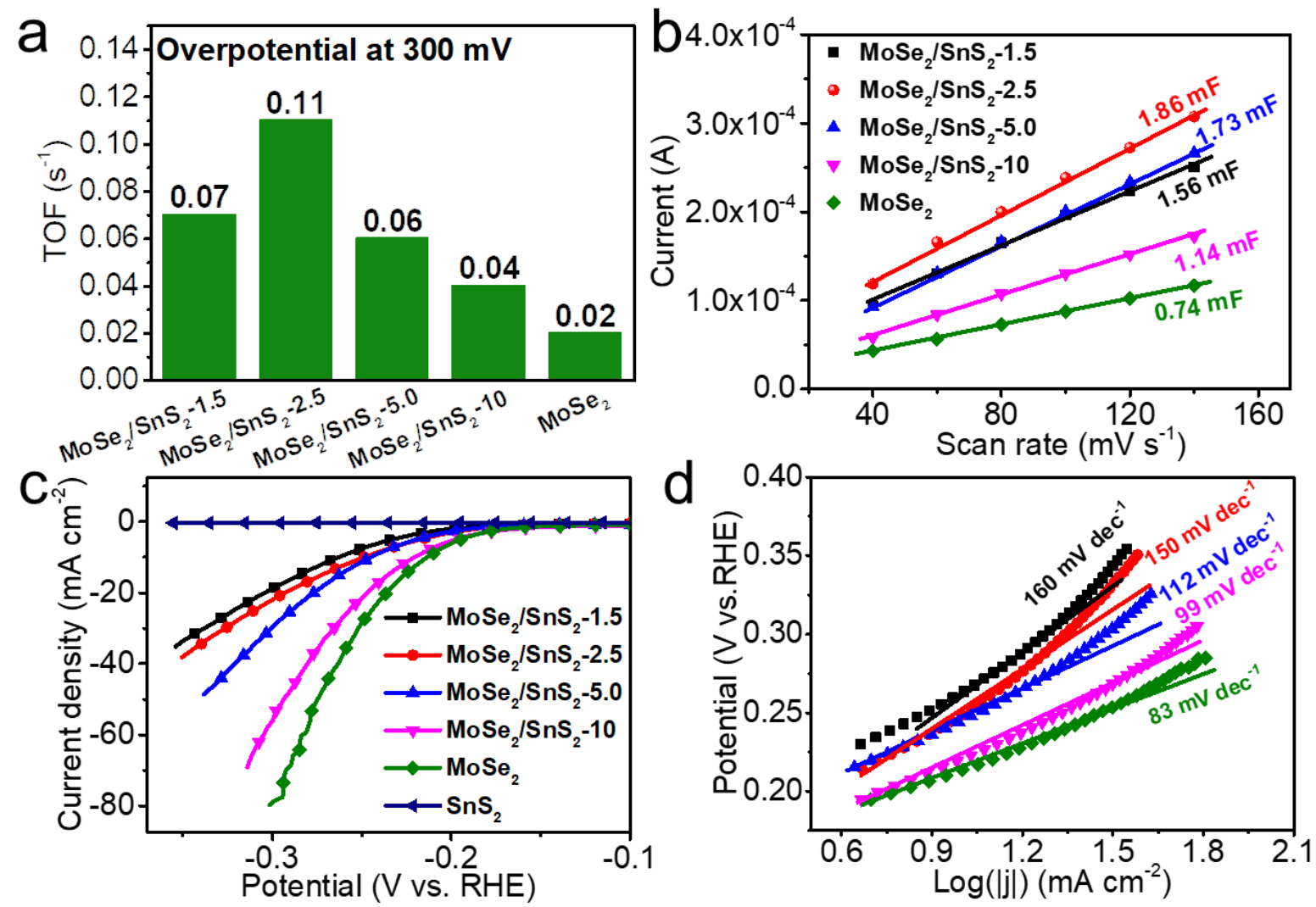

Figure 3. (a) TOFs of $\mathrm{MoSe}_{2} / \mathrm{SnS}_{2}$ heterostructures and pure $\mathrm{MoSe}_{2}$ at the overpotential of $0.3 \mathrm{~V}$ (vs. RHE); (b) Current versus scan rate measured at 0 V (vs. RHE); (c) The LSV curves and (d) Tafel plots measured in $0.5 \mathrm{M} \mathrm{H}_{2} \mathrm{SO}_{4}$. 
To further confirm the effect of incorporating $\mathrm{SnS}_{2}$ on the enhanced alkaline HER activity of the $\mathrm{MoSe}_{2} / \mathrm{SnS}_{2}$ heterostructured catalysts, the acidic HER performances of $\mathrm{MoSe}_{2} / \mathrm{SnS}_{2}$ and bare $\mathrm{MoSe}_{2}$ catalysts were tested in $0.5 \mathrm{M} \mathrm{H}_{2} \mathrm{SO}_{4}$ electrolyte. According to previously reported volcano plots, the HER kinetics of a catalyst in acidic conditions is strongly correlated with its hydrogen adsorption capability. ${ }^{[53-54]}$ In sharp contrast to the bare $\mathrm{MoSe}_{2}$, all the $\mathrm{MoSe}_{2} / \mathrm{SnS}_{2}$ heterostructures show higher overpotentials with larger Tafel slopes (Figure 3c-d). The reduced acidic catalytic activities of the $\mathrm{MoSe}_{2} / \mathrm{SnS}_{2}$ heterostructured catalysts demonstrate that the introduction of $\mathrm{SnS}_{2}$ had no positive effect on optimizing the hydrogen adsorption capability. It can be inferred that the enhanced alkaline HER activity of the $\mathrm{MoSe}_{2} / \mathrm{SnS}_{2}$ heterostructures can be mainly attributed to the accelerated water adsorption/dissociation process.

Basically, water adsorption takes place prior to water dissociation during the alkaline HER process. Therefore, to further verify the influence of $\mathrm{SnS}_{2}$ quantum dots on the alkaline HER kinetics of $\mathrm{MoSe}_{2}$, DFT calculations were conducted to determine the water adsorption energy $\left(E_{\mathrm{ad}}\right)$ of the basal planes and edges of pure $\mathrm{MoSe}_{2}, \mathrm{SnS}_{2}$, and $\mathrm{MoSe}_{2} / \mathrm{SnS}_{2}$ (Figure S7, Supporting Information). As shown in Figure 4a, the $E_{\mathrm{ad}}$ of the basal planes of $\mathrm{MoSe}_{2}$ is -0.12 $\mathrm{eV}$. After incorporating $\mathrm{SnS}_{2}$, the $E_{\mathrm{ad}}$ of the basal planes of $\mathrm{MoSe}_{2} / \mathrm{SnS}_{2}$ decreases to $-0.23 \mathrm{eV}$, suggesting that $\mathrm{SnS}_{2}$ efficiently enhances the water adsorption capability on the basal planes of $\mathrm{MoSe}_{2}$. The Mo edges, as the most stable sites for water adsorption, displays a lower $\mathrm{E}_{\mathrm{ad}}(-$ $0.82 \mathrm{eV}$ ) for $\mathrm{MoSe}_{2} / \mathrm{SnS}_{2}$ than that of $\mathrm{MoSe}_{2}(0.71 \mathrm{eV})$ (Figure 4b). Meanwhile, the Se edges with $100 \%$ and $50 \%$ Se coverage $\left(\mathrm{Se}_{100-\mathrm{eg}}\right.$ and $\left.\mathrm{Se}_{50-\mathrm{eg}}\right)$ of $\mathrm{MoSe}_{2} / \mathrm{SnS}_{2}$ heterostructures also show decreased $E_{\text {ad }}$ values relative to that of pure $\mathrm{MoSe}_{2}$. These results clearly demonstrate that the incorporation of $\mathrm{SnS}_{2}$ significantly enhances the water adsorption capability of $\mathrm{MoSe}_{2}$ in the heterostructures, which is greatly beneficial to accelerating the subsequent water dissociation kinetics. In addition, the basal planes and edges of $\mathrm{SnS}_{2}$ also show promising water 
adsorption capability (Figure S8, Supporting Information), which can further improve the water adsorption and dissociating kinetics of the $\mathrm{MoSe}_{2} / \mathrm{SnS}_{2}$ heterostructures.
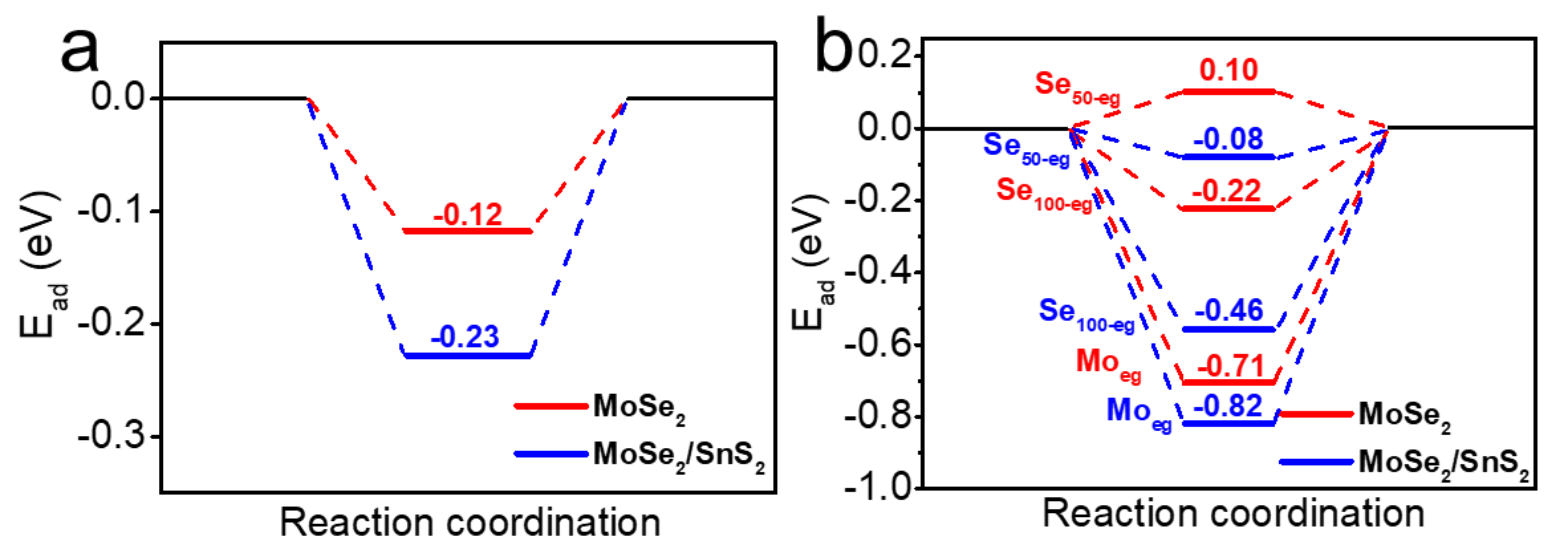

Figure 4. Water adsorption energy diagram of $\mathrm{MoSe}_{2} / \mathrm{SnS}_{2}$ and $\mathrm{MoSe}_{2}$ (a) on the basal planes and (b) on the edge sites.

$\mathrm{MoS}_{2} / \mathrm{SnS}_{2}$ heterostructures with $\mathrm{SnS}_{2}$ quantum dots decorated on the basal planes (Figure $\mathrm{S} 9$, Supporting Information), which were synthesized by a similar process to that for $\mathrm{MoSe}_{2} / \mathrm{SnS}_{2}$ heterostructures, were also evaluated as the catalysts for the alkaline HER. As shown in Figure S10a-b (Supporting Information), the alkaline HER activity of the heterostructured catalysts is also greatly enhanced after the introduction of $\mathrm{SnS}_{2}$. The optimal $\mathrm{MoS}_{2} / \mathrm{SnS}_{2}-2.5$ catalyst delivers the lowest overpotential of $343 \mathrm{mV}$ at $10 \mathrm{~mA} \mathrm{~cm}^{-2}$ with a Tafel slope of $157 \mathrm{mV} \mathrm{dec}$ 1, while pure $\mathrm{MoS}_{2}$ has an overpotential of $419 \mathrm{mV}$ at $10 \mathrm{~mA} \mathrm{~cm}{ }^{-2}$ with a Tafel slope of 216 $\mathrm{mV} \mathrm{dec}{ }^{-1}$. Similar to the case of $\mathrm{MoSe}_{2} / \mathrm{SnS}_{2}$, the acidic HER performance of the $\mathrm{MoS}_{2} / \mathrm{SnS}_{2}$ heterostructures gets worse with increasing the content of inactive $\mathrm{SnS}_{2}$ (Figure S11a-b, Supporting Information). These results demonstrate that decorating $\mathrm{SnS}_{2}$ quantum dots on basal planes to construct heterostuctures is a universal approach to promote the alkaline HER kinetics of molybdenum dichalcogenide-based catalysts.

\section{Conclusion}


In summary, molybdenum dichalcogenide heterostructures with $\mathrm{SnS}_{2}$ quantum dots decorated on the basal planes were designed and synthesized as efficient alkaline HER electrocatalysts. The optimal $\mathrm{MoSe}_{2} / \mathrm{SnS}_{2}$ heterostructured catalyst delivered a substantially lower overpotential of $285 \mathrm{mV}$ as compared with $\mathrm{MoSe}_{2}(367 \mathrm{mV})$ at $10 \mathrm{~mA} \mathrm{~cm}{ }^{-2}$. The significant improvement in alkaline HER activity is mainly due to the accelerated water adsorption/dissociation kinetics. The DFT calculations reveal that the incorporation of $\mathrm{SnS}_{2}$ can significantly improve the water adsorption capability of $\mathrm{MoSe}_{2}$, which is critical for the subsequent water dissociation process. This work opens up a new direction for the development of efficient alkaline HER electrocatalysts by engineering heterostructures.

\section{Acknowledgements}

This work was financially supported by the Australian Research Council (ARC) DECRA Grant (DE160100596), ARC Discovery Project (DP160102627), AIIM FOR GOLD Grant (2018, 2019), and UOW's Vice-Chancellor's Postdoctoral Research Fellowship Funding (X.W.). Y. C., K.R. and X. Z. are sincerely thankful for the funding support from the China Scholarship Council (CSC). We are grateful to the High Performance Computing Center of Nanjing University for providing the IBM Blade cluster system. We also acknowledge use of the facilities within the UOW Electron Microscopy Centre and Dr. Tania Silver for her critical reading.

\section{References}

[1] S. Chu, A. Majumdar, Nature 2012, 488, 294-303.

[2] J. Wang, K. Li, H.-x. Zhong, D. Xu, Z.-1. Wang, Z. Jiang, Z.-j. Wu, X.-b. Zhang, Angew. Chem. Int. Ed. 2015, 127, 10676-10680.

[3] P. Chen, T. Zhou, M. Zhang, Y. Tong, C. Zhong, N. Zhang, L. Zhang, C. Wu, Y. Xie, Adv. Mater. 2017, 29, 1701584-1701590.

[4] Z. Gao, J. Qi, M. Chen, W. Zhang, R. Cao, Electrochim. Acta 2017, 224, 412-418.

[5] J. Mahmood, M. A. R. Anjum, S.-H. Shin, I. Ahmad, H.-J. Noh, S.-J. Kim, H. Y. Jeong, J. S. Lee, J.-B. Baek, Adv. Mater. 2018, 30, 1805606-1805613.

[6] J. A. Turner, Science 2004, 305, 972-974.

[7] D. Strmcnik, M. Uchimura, C. Wang, R. Subbaraman, N. Danilovic, D. van der Vliet, A. P. Paulikas, V. R. Stamenkovic, N. M. Markovic, Nat. Chem. 2013, 5, 300-306. 
[8] Y. Jiao, Y. Zheng, M. Jaroniec, S. Z. Qiao, Chem. Soc. Rev. 2015, 44, 2060-2086.

[9] M.-S. Balogun, W. Qiu, H. Yang, W. Fan, Y. C. Huang, G.-R. Li, H. Ji, Y. Tong, Energy Environ. Sci. 2016, 9, 3411-3416.

[10] J.-X. Feng, S.-Y. Tong, Y. Tong, G.-R. Li, J. Am. Chem. Soc. 2018, 140, 5118-5126.

[11] Y. Shenghua, Z.-X. Shi, J.-X. Feng, Y.-X. Tong, G.-R. Li, Angew.Chem. Int. Ed. 2018, 57, 2672-2676.

[12] W. Anliang, H. Xu, G.-R. Li, ACS Energy Lett. 2016, 1, 445-453.

[13] J. Feng, H. Xu, Y.-T. Dong, X.-F. Lu, Y.-X. Tong, G.-R. Li, Angew.Chem. Int. Ed. 2017, 56, 2960-2964.

[14] F. Li, G. Han, H.-J. Noh, Y. Lu, J. Xu, Y. Bu, Z. Fu, J.-B. Baek, Angew. Chem. Int. Ed. 2018, 57, 14139-14143.

[15] J. Mahmood, F. Li, S.-M. Jung, M. S. Okyay, I. Ahmad, S.-J. Kim, N. Park, H. Y. Jeong, J.-B. Baek, Nat. Nanotechnol. 2017, 12, 441-446.

[16] Z. Dai, H. Geng, J. Wang, Y. Luo, B. Li, Y. Zong, J. Yang, Y. Guo, Y. Zheng, X. Wang, Q. Yan, ACS Nano 2017, 11, 11031-11040.

[17] Z.-L. Wang, X.-F. Hao, Z. Jiang, X.-P. Sun, D. Xu, J. Wang, H.-X. Zhong, F.-L. Meng, X.-B. Zhang, J. Am. Chem. Soc. 2015, 137, 15070-15073.

[18] M. G. Walter, E. L. Warren, J. R. McKone, S. W. Boettcher, Q. Mi, E. A. Santori, N. S. Lewis, Chem. Rev. 2010, 110, 6446-6473.

[19] Y. Chen, Q. Zhou, G. Zhao, Z. Yu, X. Wang, S. X. Dou, W. Sun, Adv. Funct. Mater. 2017, 28, 1705583-1705590.

[20] B. M. Hunter, H. B. Gray, A. M. Müller, Chem. Rev. 2016, 116, 14120-14136.

[21] Z. Chen, Y. Song, J. Cai, X. Zheng, D. Han, Y. Wu, Y. Zang, S. Niu, Y. Liu, J. Zhu, X. Liu, G. Wang, Angew. Chem. Int. Ed. 2018, 57, 5076-5080.

[22] C. Tan, H. Zhang, Chem. Soc. Rev. 2015, 44, 2713-2731.

[23] X. Sun, J. Dai, Y. Guo, C. Wu, F. Hu, J. Zhao, X. Zeng, Y. Xie, Nanoscale 2014, 6, 8359-8367.

[24] Y. Zhang, Q. Zhou, J. Zhu, Q. Yan, S. X. Dou, W. Sun, Adv. Funct. Mater. 2017, 27, 1702317.

[25] K. Tang, X. Wang, Q. Li, C. Yan, Adv. Mater. 2018, 30, 1704779-1704786.

[26] K. K. Ghuman, S. Yadav, C. V. Singh, J. Phys. Chem. C 2015, 119, 6518-6529.

[27] C. Tsai, K. Chan, F. Abild-Pedersen, J. K. Nørskov, Phys. Chem. Chem. Phys. 2014, 16, 13156-13164.

[28] Y. Shi, Y. Zhou, D. Yang, W. Xu, C. Wang, F. Wang, J. Xu, X. Xia, H. Chen, J. Am. Chem. Soc. 2017, 139, 15479-15485.

[29] N. Xue, P. Diao, J. Phys. Chem. C 2017, 121, 26686-26697.

[30] H. Shu, D. Zhou, F. Li, D. Cao, X. Chen, ACS Appl. Mater. Inter. 2017, 9, 4268842698.

[31] O. Lehtinen, H.-P. Komsa, A. Pulkin, M. B. Whitwick, M.-W. Chen, T. Lehnert, M. J. Mohn, O. V. Yazyev, A. Kis, U. Kaiser, A. V. Krasheninnikov, ACS Nano 2015, 9 , 3274-3283.

[32] Y. Yin, Y. Zhang, T. Gao, T. Yao, X. Zhang, J. Han, X. Wang, Z. Zhang, P. Xu, P. Zhang, X. Cao, B. Song, S. Jin, Adv. Mater. 2017, 29, 1700311-1700319.

[33] K. Xu, H. Ding, M. Zhang, M. Chen, Z. Hao, L. Zhang, C. Wu, Y. Xie, Adv. Mater. 2016, 29, 1606980-1606986.

[34] G. Chen, T. Wang, J. Zhang, P. Liu, H. Sun, X. Zhuang, M. Chen, X. Feng, Adv. Mater. 2018, 30, 1706279-1706286.

[35] J. Zhang, T. Wang, P. Liu, Z. Liao, S. Liu, X. Zhuang, M. Chen, E. Zschech, X. Feng, Nat. Commun. 2017, 8, 15437-15445. 
[36] M. Lao, K. Rui, G. Zhao, P. Cui, X. Zheng, S. X. Dou, W. Sun, Angew. Chem. Int. Ed. 2019, 58, 5432-5437.

[37] G. Zhao, K. Rui, S. X. Dou, W. Sun, Adv. Funct. Mater. 2018, 28, 1803291.

[38] G. Zhao, Y. Lin, K. Rui, Q. Zhou, Y. Chen, S. X. Dou, W. Sun, Nanoscale 2018, 10, 19074-19081.

[39] G. Zhao, P. Li, K. Rui, Y. Chen, S. X. Dou, W. Sun, Chem. Eur. J. 2018, 24, 1115811165.

[40] C. Lei, Y. Wang, Y. Hou, P. Liu, J. Yang, T. Zhang, X. Zhuang, M. Chen, B. Yang, L. Lei, C. Yuan, M. Qiu, X. Feng, Energy Environ. Sci. 2019, 12, 149-156.

[41] B. Song, S. Jin, Joule 2017, 1, 220-221.

[42] B. Zhang, J. Liu, J. Wang, Y. Ruan, X. Ji, K. Xu, C. Chen, H. Wan, L. Miao, J. Jiang, Nano Energy 2017, 37, 74-80.

[43] J. Yang, C. Wang, H. Ju, Y. Sun, S. Xing, J. Zhu, Q. Yang, Adv. Funct. Mater. 2017, 27, 1703864-1703874.

[44] X. Zhou, Y. Liu, H. Ju, B. Pan, J. Zhu, T. Ding, C. Wang, Q. Yang, Chem. Mater. 2016, 28, 1838-1846.

[45] J. Zhang, T. Wang, P. Liu, S. Liu, R. Dong, X. Zhuang, M. Chen, X. Feng, Energy Environ. Sci. 2016, 9, 2789-2793.

[46] X.-Y. Yu, Y. Feng, Y. Jeon, B. Guan, X. W. Lou, U. Paik, Adv. Mater. 2016, 28, 9006-9011.

[47] C. C. McCrory, S. Jung, J. C. Peters, T. F. Jaramillo, J. Am. Chem. Soc. 2013, 135, 16977-16987.

[48] C. Ataca, S. Ciraci, Phys. Rev. B 2012, 85, 195410.

[49] Y.-C. Chen, A.-Y. Lu, P. Lu, X. Yang, C.-M. Jiang, M. Mariano, B. Kaehr, O. Lin, A. Taylor, I. D. Sharp, L.-J. Li, S. S. Chou, V. Tung, Adv. Mater. 2017, 29, 17038631703874.

[50] H. Li, C. Tsai, A. L. Koh, L. Cai, A. W. Contryman, A. H. Fragapane, J. Zhao, H. S. Han, H. C. Manoharan, F. Abild-Pedersen, J. K. Nørskov, X. Zheng, Nat. Mater. 2015, $15,48-54$.

[51] S. Sun, H. Li, Z. J. Xu, Joule 2018, 2, 1024-1027.

[52] Y. Wu, X. Liu, D. Han, X. Song, L. Shi, Y. Song, S. Niu, Y. Xie, J. Cai, S. Wu, J. Kang, J. Zhou, Z. Chen, X. Zheng, X. Xiao, G. Wang, Nat. Commun. 2018, 9, 1425.

[53] T. F. Jaramillo, K. P. Jørgensen, J. Bonde, J. H. Nielsen, S. Horch, I. Chorkendorff, Science 2007, 317, 100-102.

[54] J. Greeley, T. F. Jaramillo, J. Bonde, I. Chorkendorff, J. K. Nørskov, Nat. Mater. 2006, 5, 909. 